\title{
A Comparative Study of Rapid Salmonella-IgM Test (Typhi-dot M) and Widal Test in the Diagnosis of Enteric Fever in a Tertiary Hospital
}

\author{
V. Mallikarjun Rao* \\ Department of Microbiology, Shadan Institute of Medical Sciences and Research Centre, \\ Hyderabad, Telangana, India \\ *Corresponding author
}

\section{A B S T R A C T}

\section{Keywords}

Enteric fever, Blood

culture, Rapid

salmonella-IgM test,

Typhidot-IgM, Widal test

Article Info

Accepted:

23 March 2018

Available Online:

10 April 2018
Typhoid fever is a noted cause of morbidity worldwide and a major public health problem in India and other developing countries. An early and accurate diagnosis is essential for a prompt and effective treatment. The present study was conducted with the aim of comparing the efficacy of rapid Salmonella-IgM test (Typhi-dot M) and Widal test in both blood culture positive and negative patients in the diagnosis of enteric fever in a tertiary hospital. A total 794 patients serum samples were tested by rapid Salmonella-IgM test (Typhi-dot M), Widal test and blood culture. The sensitivity, specificity, positive predictive value (PPV) and negative predictive value (NPV) of Typhidot-IgM test, considering blood culture positive for $S$. typhi as gold standard, were 93.04\%, 80.08\%, $74.14 \%$ and $94.93 \%$ respectively and $36.09 \%, 58.53 \%, 34.82 \%$ and $59.87 \%$ respectively for Widal test. Rapid Salmonella-IgM test (Typhi-dot M) is significantly more sensitive and specific as compared to Widal test in the diagnosis of enteric fever. It can be utilized in conjunction with blood culture for diagnosis of enteric fever.

\section{Introduction}

Typhoid (enteric) fever caused by Salmonella enterica serovar Typhi (S. typhi) is a noted cause of morbidity worldwide with an estimated 21.7 million cases, the bulk of the burden being borne by India, South and Central America and subSaharan Africa, all with growing population and poor sanitary conditions (Banerjee et al., 2014). Antibiotic resistance is a challenge for effective treatment of typhoid and is likely to become increasingly problematic with the spread of multi-drug resistant strains as in India, most of the infections by Salmonella enterica serotype Typhi are diagnosed clinically without proper laboratory evidence and consequently treated presumptively with antibiotics (Olsen et al., 2004; Wain et al., 2014).

Blood culture, the gold standard for diagnosing enteric fever (Parry et al., 2002) provides a definitive diagnosis in 73-97 per cent cases prior to medications but excessive antibiotic use has reduced this isolation rate to 40- 60 per cent (Wain et al., 2014). The Widal test has been and is still being widely used for the diagnosis of typhoid fever, owing to the fact that no other sero-diagnostic test of 
sufficient sensitivity and specificity along with cost-effectiveness has been developed especially in typhoid endemic regions. A paired serum sample with a fourfold rise in titer is needed for a meaningful result.

It takes more than 1 week for the significant titers of antibodies to appear (Olopoenia and King, 2000). Accurate diagnosis of enteric fever at an early stage also helps to identify individuals who may serve as potential carriers, who may be responsible for acute enteric fever outbreaks (Threlfall, 2005).

Several commercial rapid diagnostic tests namely Typhidot and Tubex have also shown sensitivity and specificity of 70 and 80 per cent, respectively in most of the surveillance studies worldwide, apart from being costlier than the agglutination tests (Ley et al.,) Initial studies done in Asian countries like Malaysia, Indonesia, Philippines, Pakistan, Bangladesh and India have shown variable sensitivity (7395\%) and specificity (68-95\%) of these tests (Sherwal et al., 2004; Jesudason and Sivakumar, 2006; Narayanappa et al., 2010; Bhutta and Mansurrali, 1999). However, there are relatively few studies comparing these rapid diagnostic tests with widal test using blood culture as the gold standard.

Thus, the present study was conducted with the aim of comparing the efficacy of rapid Salmonella-IgM test (Typhi-dot M) and Widal test in both culture positive and culture negative patients in the diagnosis of enteric fever in a tertiary hospital.

\section{Materials and Methods}

The present study was a cross sectional study; the period of the study was six months, from June 2017 to December 2017. It was conducted in department of Microbiology, Bhaskar Medical College and hospital; Yenkapally. It is a tertiary teaching hospital around $30 \mathrm{~km}$ from Hyderabad, Telangana.
All patients who were admitted in the hospital or attended the outpatient (OPD) department or emergency department with symptoms and signs of typhoid fever during the study period were the study participants. Approval was taken from Institutional ethics committee prior to commencement of the study.

In all patients following a detailed clinical examination and other routine investigations; the following tests were done blood culture, Rapid Salmonella-IgM test (Typhidot-IgM) and Widal test. The study was finally conducted on 794 patients. In addition to blood culture positive cases, significance of positive Salmonella-IgM tests among blood culture-negative cases was also assessed.

\section{Blood culture}

Blood culture was done according to the standard procedures using the BACTEC system and surveyed the results after 5 days. If there was any growth, colonies were subcultured to blood agar. Isolates were Gram stained and identified by standard biochemical methods. Serotyping was performed by using agglutination with Salmonella $\mathrm{O}, \mathrm{H}$, and $\mathrm{Vi}$ antisera. If there was no growth after 10 days, the culture was considered negative.

\section{Widal test}

Widal testing was done by using the Sanofi qualitative agglutination test kits (Bio-Rad). Serum was serially diluted, starting at $1 / 10$, in physiological saline and then further diluted $1 / 10$ in suspensions containing serotype Typhi $\mathrm{O}$ and $\mathrm{H}$ antigens, separately.

The classical technique with incubation in which the tubes were incubated in a $37^{\circ} \mathrm{C}$ water bath for $2 \mathrm{~h}$ for $\mathrm{H}$ suspensions and at room temperature overnight for $\mathrm{O}$ suspensions; if agglutination was visible, the results were considered positive. 
Typhidot-IgM (Salmonella typhi specific IgM antibody assay)

It is a qualitative antibody detection test based on dot enzyme immunoassay technique, to detect the presence of $\operatorname{IgM}$ antibodies against a specific antigen on the outer membrane of $S$. typhi.

The test was carried out as per the manufacturer's instructions (Malaysian Biodiagnostics Research Sdn. Bhd, Selangor Darul Ehsan, Malaysia). The Typhidot-M test measures IgM against the same $50 \mathrm{kDa}$ antigen in the same dot-blot format after removal of the total IgG.

\section{Results and Discussion}

Of a total of 794 patients, S. typhi was isolated from blood culture in $302(38.03 \%)$, in the remaining 492 cases the blood culture was found to be negative.

Among the blood culture negative patients 37 patients had an alternate diagnosis finally and were treated accordingly. Among 794 patients; Widal test was positive in $204(25.69 \%)$ patients and Typhidot-M test was positive in 379 cases $(47.73 \%)$.

Comparison of Typhidot $\mathrm{M}$ and Widal test with Blood Culture is detailed in Table 1.

\section{Typhidot-M}

Of a total of 794 patients, S. typhi was isolated from blood culture in $302(38.03 \%)$ and Typhidot-M test was positive in 379 (47.73\%) patients.

Among blood culture positive cases out of 302 patients, 281(93.04) were also positive by Typhidot-IgM antibody test. The sensitivity, specificity, positive predictive value (PPV) and negative predictive value (NPV) of
Typhidot-IgM test, considering blood culture positive for $S$. typhi as gold standard, were $93.04 \%, 80.08 \%, 74.14 \%$ and $94.93 \%$ respectively (Table 2).

\section{Widal test}

Of a total of 794 patients, Widal test was positive in $379(47.73 \%)$ patients. Among 302 patients whose blood culture was positive for S. typhi, Widal test was also positive in 109 (36.09\%) patients. The sensitivity, specificity, positive predictive value (PPV) and negative predictive value (NPV) of Widal test, considering blood culture positive for S. typhi as gold standard, were $36.09 \%, 58.53 \%$, $34.82 \%$ and $59.87 \%$ respectively (Table 2 ).

The sensitivity, specificity, and positive and negative predictive value of Typhidot-M and Widal test is compared in table 2.

In patients with fever less than 7 days, $(\mathrm{n}=213)$, Typhi-dot $\mathrm{M}$ was positive in 192 (90.14\%) patients. Widal test was positive in $62(29.11 \%)$ patients. This difference in positivity among the two tests was highly significant statistically. $(\mathrm{P}<0.001)$ In patients with fever more than 7 days there was no statistically significant difference in positivity $(\mathrm{P}>0.05)$.

In the present study, the sensitivity of Typhidot-M was determined to be $93.04 \%$, which is comparable to that reported by other similar studies (Wain et al., 2014; Narayanappa et al., 2010; Bhutta and Mansurrali, 1999; Gopalakrishna et al., 2002; Dutta et al., 2006). Sensitivity indicates the percentage of patients with a positive test result who are correctly diagnosed with disease. Specificity indicates the percentage of patients who are correctly identified as not having disease. Thus, Typhidot- $\mathrm{M}$ is a sensitive test with few false negatives, which is one of the criteria of an ideal diagnostic test. 
Table.1 Comparison of Typhidot $\mathrm{M}$ and Widal test with blood culture

\begin{tabular}{|c|c|c|c|c|}
\hline Blood culture & \multicolumn{2}{|c|}{ Typhidot -M (N=794) } & \multicolumn{2}{c|}{ Widal N=794 } \\
\hline & Positive & Negative & Positive & Negative \\
\hline Positive N=302 & 281(93.04) & 21(6.96) & 109(36.09) & $193(63.91)$ \\
\hline Negative $\mathbf{N = 4 9 2}$ & 98(19.92) & 394(80.08) & 204(41.46) & 288(58.54) \\
\hline
\end{tabular}

Figures in parenthesis indicate percentages

Table.2 Comparison of sensitivity, specificity, positive and negative predictive value of Typhidot-M and Widal test

\begin{tabular}{|l|c|c|}
\hline & Typhidot-M & Widal test \\
\hline Sensitivity (\%) & 93.04 & 36.09 \\
\hline Specificity (\%) & 80.08 & 58.53 \\
\hline PPV (\%) & 74.14 & 34.82 \\
\hline NPV (\%) & 94.93 & 59.87 \\
\hline
\end{tabular}

In a recent systematic review and metaanalysis by Wijedoru et al., (2015) twenty two Typhidot studies (Typhidot, Typhidot-M, and TyphiRapid-Tr02), showed an average sensitivity of $84 \%$ and specificity was $79 \%$, which is lesser compared to the present study probably due to pooled results. In a similar study by Prasad et al., (2015) sensitivity, specificity, positive predictive value (PPV) and negative predictive value (NPV) of the Typhidot-IgM test was $97.29 \%, 97.40 \%$, $98.18 \%$, $96.15 \%$ respectively, which is comparable to the present study.

Twenty-one patients who were positive on blood culture were negative by Typhidot-M. All patients had presented with fever of duration more than seven days; probably due to of decreasing levels of IgM and masking of IgM by $\operatorname{IgG}$ contributing to a negative Typhidot-M test result. This could have been avoided with concomitant use of Typhidot (IgG) (Bhutta and Mansurrali, 1999; Gopalakrishna et al., 2002).

A total of $98(19.92 \%)$ patients were detected as false positive by Typhi-Dot $M$ in the present study. Similar findings are reported by other studies (Bhutta and Mansurrali, 1999;
Prasad et al., 2015) Blood culture has an estimated sensitivity of between $40 \%$ and 80\% (Fadeel et al., 2004), with a more recent systematic review estimating sensitivity to be around 60\% (McKinnon and Abdool Karim, 2014) and was the reference standard in the present study. Also in 31(31.63\%) of these patients the duration of fever was more than 7 days which could have resulted in a negative blood culture. Among the 98 patients, 19 (19.38\%) were clinically diagnosed to have enteric fever supported by leucocyte counts, C reactive protein and responded to IV Ceftriaxone.

In the present study, the sensitivity, specificity, positive predictive value (PPV) and negative predictive value (NPV) of Widal test were $36.09 \%, 58.53 \%, 34.82 \%$ and $59.87 \%$ respectively which is significantly lesser than that of Typhidot $\mathrm{M}(\mathrm{P}<0.001)$ and is comparable to other similar studies (Narayanappa et al., 2010; Wijedoru et al., 2017). In their study Dutta et al., (2006) reported $58 \%$ sensitivity, $85 \%$ specificity, $69 \%$ PPV, and $77 \%$ NPV for Widal test and the sensitivity, specificity, PPV, and NPV of Typhidot was not found to be better than Widal test; which is contrary to the finding of 
the present study probably; due to difference in study setting and duration of fever among the patients. Ideally, the Widal test should be done on paired sera to detect an increase in the agglutination titer.

However, to aid treatment decisions before convalescent samples can be obtained, single acute-phase serum sample are done. The results from a single sample are difficult to interpret due high false positive results.

The role of the Widal test is controversial because the sensitivity, specificity, and predictive values vary considerably between geographical areas (Mogasale et al., 2016) Despite these shortcomings of both sensitivity and specificity, because the Widal test is simple and inexpensive, it is still widely used as a diagnostic test (McKinnon and Abdool Karim, 2014).

A clinically useful test requires high values for both sensitivity and specificity. Typhidot$M$ is simple, sensitive, specific early, rapid and requires minimal operator training. Comparatively higher cost and cold storage requirement for test reagents are among the limitations.

The search for the ideal rapid diagnostic test for enteric fever continues. Research into developing a better reference standard for the diagnosis of enteric fever in both adults and children is needed (McKinnon and Abdool Karim, 2014). Alternative biomarkers of acute enteric fever, such as metabolomic profiles (McKinnon and Abdool Karim, 2014) could form the basis of new groups of RDTs.

Rapid Salmonella-IgM test (Typhi-dot $M$ ) is more sensitive and specific test as compared to Widal test which is the most commonly used test; in the diagnosis of enteric fever. It can be utilized in conjunction with blood culture for diagnosis of enteric fever.

\section{References}

Banerjee T, Shukla BN, Filgona J, Anupurba S, Sen MR. Trends of typhoid fever seropositivity over ten years in North India. Indian J Med Res. 2014; 140: 310-3.

Bhutta ZA, and Mansurrali N. Rapid serologic diagnosis of Pediatric typhoid fever in an endemic area: A prospective comparative evaluation of two dot enzyme immunoassay and the Widal test. Am J Trop Med Hyg 1999; 61: 654.

Dutta S, Dipika S, Byomkesh M, Bhaswati S, Deb AK, Jacqueline LD, et al., Evaluation of new generation serologic tests for the diagnosis of typhoid fever: data from a community based surveillance in Calcutta, India. Diagn Microbiol Infect Dis 2006; 56: 359-365.

Fadeel MA, Crump JA, Mahoney FJ, Nakhla IA, Mansour AM, Reyad B, et al., Rapid diagnosis of typhoid fever by enzyme-linked immunosorbent assay detection of Salmonella serovar Typhi antigens in urine. American Journal of Tropical Medicine and Hygiene. 2004; 70(3): 323-8. 5668360.

Gopalakrishna V, Sekhar WY, Soo EH, Vinsent RA, Devi S. Typhoid fever in Kuala Lumpur and a comparative evaluation of two commercial diagnostic kits for the detection of antibodies to Salmonella typhi. Singapore Med J 2002; 43: 354-358.

Jesudason MV, and Sivakumar S. Prospective evaluation of a rapid diagnostic test Typhidot ${ }^{\circledR}$ for typhoid fever. Indian $\mathbf{J}$ Med Res 2006; 123: 513-6.

Ley B, Mtove G, Thriemer K, Amos B, von Seidlein L, Hendriksen I, et al., Evaluation of the Widal tube agglutination test for the diagnosis of typhoid fever among children admitted 
McKinnon LR, and Abdool Karim Q. Honing in on enteric fever. eLife. 2014; 3: $\mathrm{e} 03545$.

Mogasale V, Ramani E, Mogsale VV, Park JY. What proportion of Salmonella typhi cases are detected by blood culture? A systematic literature review. Annals of Clinical Microbiology and Antimicrobials. 2016; 15(1): 32. DOI: 10.1186/s12941-0160147-z.

Narayanappa D, Sripathi R, Jagdishkumar K, Rajani HS. Comparative study of dot enzyme immunoassay (Typhidot-M) and Widal test in the diagnosis of typhoid fever. Indian Pediatr 2010; 47: 331-3.

Olopoenia LA, and King AL. Widal agglutination test - 100 years later: still plagued by controversy. Postgrad Med J 2000; 76: 80-4.

Olsen SJ, Pruckler J, Bibb W, Than NTm, Trinh TM, Minh NT, et al., Evaluation of rapid diagnostic tests for typhoid fever. J Clin Microbiol 2004; 42: 1885 9.
Parry CM, Hien TT, Dougan G, White NJ, Farrar JJ. Typhoid fever. N Engl J Med 2002; 347: 1770-82.

Prasad K J, Oberoi J K, Goel N, Wattal C. Comparative evaluation of two rapid Salmonella-IgM tests and blood culture in the diagnosis of enteric fever. Indian J Med Microbiol 2015; 33: 237-42

Sherwal BL, Dhamija RK, Randhawa VS, Jais M, Kaintura A, Kumar M. A comparative study of Typhidot $M$ and Widal test in patients of typhoid fever. J Indian Acad Clin Med 2004; 5: 244-50.

Threlfall EJ. Salmonella. In: Borriello SP, Murray PR, Funke G (editors). Topley and Wilson's Microbiology and Microbial Infections Bacteriology. Vol 2. $10^{\text {th }}$ ed. Ch. 54. Hodder Arnold, London: ASM press; 2005. p. 1398-434

Wain J, Hendriksen RS, Mikoleit ML, Keddy KH, Ochiai RL (2014) Typhoid fever. The Lancet.

Wijedoru L, Mallett S, Parry CM. Rapid diagnostic tests for typhoid and paratyphoid (enteric) fever. The Cochrane Database of Systematic Reviews. 2017; (5): CD008892.

\section{How to cite this article:}

Mallikarjun Rao, V. 2018. A Comparative Study of Rapid Salmonella-IgM Test (Typhi-dot M) and Widal Test in the Diagnosis of Enteric Fever in a Tertiary Hospital. Int.J.Curr.Microbiol.App.Sci. 7(04): 2897-2902. doi: https://doi.org/10.20546/ijcmas.2018.704.330 